RU-96-73

\title{
Singularities, Gauge Dynamics, and Nonperturbative Superpotentials in String Theory
}

\author{
Shamit Kachru and Eva Silverstein ${ }^{\dagger}$ \\ Department of Physics and Astronomy \\ Rutgers University \\ Piscataway, NJ 08855
}

\begin{abstract}
We describe a class of $4 \mathrm{~d} \mathrm{~N}=1$ compactifications of the $S O(32)$ heterotic/type I string theory which are destabilized by nonperturbatively generated superpotentials. In the type I description, the destabilizing superpotential is generated by a one instanton effect or gaugino condensation in a nonperturbative $S U(2)$ gauge group. The dual, heterotic description involves destabilization due to worldsheet instanton or half worldsheet instanton effects in the two cases. A genericity argument suggests that a (global) supersymmetrybreaking model of Intriligator and Thomas might be typical in a class of string theory models. Our analysis also suggests that the tensionless strings which arise in the $E_{8} \times E_{8}$ theory in six dimensions when an instanton shrinks to zero size should, in some cases, have supersymmetry breaking dynamics upon further compactification to four dimensions. We provide explicit examples, constructed using F-theory, of the two cases of dynamically generated superpotentials.
\end{abstract}

$08 / 96$

† kachru@physics.rutgers.edu, evas@physics.rutgers.edu 


\section{Introduction}

A long-standing problem in string theory has been the tremendous degeneracy of vacuum states. Although in the case of vacua with extended supersymmetry this problem appears to persist nonperturbatively, one expects that many of the classical ground states preserving $4 \mathrm{~d} N=1$ supersymmetry should be lifted by nonperturbative effects. The ultimate hope is of course to find models with nonperturbative superpotentials which leave minima with broken supersymmetry and vanishing vacuum energy.

One potential source of nonperturbative superpotentials in string theory is strong infrared dynamics. Supersymmetric QCD with $N_{f} \leq N_{c}-1$ [1] is a prototypical example of a theory where nonperturbative dynamics generates a superpotential. In this paper, we argue that the nonperturbative superpotentials of SUSY QCD and pure SUSY YangMills also make an appearance in four dimensional $S O(32)$ string compactifications on $K 3$ fibrations. The dynamics of the $N_{c}=N_{f}=2$ theory has already been used in [2] to explain poles in the Yukawa couplings of conventional $\mathrm{SO}(32)$ string compactifications on Calabi-Yau threefolds, while the $N_{f}=0$ theory made an appearance in an $\mathrm{N}=1 / \mathrm{N}=0$ dual pair proposed in [3]. Here, we use similar ideas to describe type I string compactifications and F-theory compactifications where a destabilizing superpotential is generated by

(a) instanton effects in a nonperturbative $S U(2)$ gauge group with two massless doublets, or

(b) gaugino condensation in a nonperturbative pure $S U(2)$ gauge theory.

Both (a) and (b) can occur at small instanton singularities in the classical moduli space. The superpotentials generated in both cases are nonperturbative in the type I string coupling, and map to worldsheet instanton and half worldsheet instanton effects in the heterotic string.

The destabilizing superpotentials which we find can be traced to the presence of singularities, which arise at codimension 1 in the moduli space of vacua in case (a) and

which are frozen into the compactification in case (b). In the type I description the singularities involve degenerations of the gauge bundle over one point in each $K 3$ fiber. Such singularities can be interpreted as requiring the presence of (wrapped) five-branes in the vacuum, as in [2]. In a dual F-theory description, the analogous singularities are given by elliptic fourfolds with $A_{1}$ degenerations of the elliptic fiber over a surface. While to a naive perturbative string theorist generically singular vacua which exhibit a superpotential of type (b) would have seemed problematic as compactification spaces, it is clear in light of 
recent progress that such vacua are perfectly sensible, and simply involve suitable wrapped D-branes. In fact, in some sense such vacua should be generic, in that we no longer need to impose conditions that ensure that the conformal field theory alone (without inclusion of D-branes) is nonsingular.

In many standard compactifications of heterotic/type I string theory, one has unbroken nonabelian subgroups of the perturbative gauge group which are generically present, sometimes without charged matter. It is worth emphasizing here that the phenomena we are studying do not depend on any features of an unbroken perturbative gauge group. The nonperturbative gauge groups we are studying arise purely from local degenerations of the string compactification, and can occur in the absence of any unbroken perturbative gauge symmetry. In fact in the heterotic string, they lead to effects which occur at the level of worldsheet instantons and so are stronger (at weak string coupling) than any nonperturbative effects associated with the perturbative gauge groups.

\section{Singularities, Gauge Dynamics, and Tensionless Strings}

In this paper, we study singularities in heterotic/type I compactifications on threefolds $M$ which are $K 3$ fibrations. In choosing such a compactification, one must also specify a stable, holomorphic vector bundle $V \rightarrow M$ with suitable properties [4].

As discussed in [2], degenerations of $V$ which occur at a point in the generic (smooth) $K 3$ fiber of $M$ (and hence occur over a rational curve $C$ in $M$ ) are equivalent to some configuration of "small instantons" or D 5-branes [5] wrapped on $C$. 1 We focus here on configurations involving a single small instanton. By taking a limit where $C$ is large, one can derive the spectrum of the four-dimensional theory from the spectrum of the sixdimensional compactification on the fiber $K 3$. The $4 \mathrm{~d}$ theory is a twisted version of the sixdimensional theory compactified on $C$, where the twist preserves $4 \mathrm{~d} N=1$ supersymmetry. The zero modes of the relevant Dirac operators on $C$ yield the massless $4 \mathrm{~d}$ spectrum.

The spectrum in six dimensions, for a single small instanton, consists of $S U(2)$ gauge theory with 32 doublets of $S U(2)$ [5]. A $4 \mathrm{~d} \mathrm{~N}=1 S U(2)$ gauge multiplet survives the reduction of the $6 \mathrm{~d} N=1$ gauge multiplet to four dimensions. Determining the spectrum of charged matter fields which survive involves more calculation: $2 \times \operatorname{rank}(\mathrm{V})$ of the doublets

1 We are assuming the $K 3$ fibration admits section(s) and choosing one so we can discuss the base of the fibration $C$. 
couple to the connection on $V$ and $V^{*}$ while the rest couple to a trivial gauge connection. Any holomorphic bundle $V$ splits as a direct sum of line bundles $\sum_{i} \mathcal{O}\left(n_{i}\right)$ when restricted to a rational curve $C$.2 As discussed in [2], the massless spectrum which survives at the singularity depends crucially on the splitting type of $V$ when restricted to $C$ - the surviving doublets correspond to elements of $H^{0}\left(\mathcal{O}\left(n_{i}-1\right)\right)$ and $H^{0}\left(\mathcal{O}\left(-n_{i}-1\right)\right)$.

In [2], we studied a bundle which split as $\mathcal{O}(2) \oplus \mathcal{O}(-2) \oplus \mathcal{O}(0)$. The resulting spectrum, combined with a tree-level superpotential, dynamically generated a pole in the Yukawa coupling of charged generations. In the remainder of this section, we describe in general terms two classes of singularities of the $4 \mathrm{~d} N=1$ vacua which lead to a destabilizing superpotential. In the next section, we discuss in some detail examples (derived from F-theory) of both types.

\subsection{Instanton Destabilization}

We expect one type of nonperturbative superpotential to be generated in the following circumstances. Suppose there is a singular locus at codimension one in the moduli space of vacua, where $V$ is singular over a point on the generic $K 3$ fiber. Furthermore, suppose that $V$ splits as

$$
\left.V\right|_{C}=\mathcal{O}(1) \oplus \mathcal{O}(-1) \oplus \mathcal{O}(0) \oplus \ldots
$$

where ... denotes more trivial factors. At such singularities, one obtains an extra nonperturbative $S U(2)$ gauge group with two massless doublets. As described many years ago [1], instanton effects destabilize the moduli space of classical vacua of this theory. If we denote by $\Lambda$ the scale of the $S U(2)$, and by $V_{12}=\epsilon_{\alpha \beta} d_{1}^{\alpha} d_{2}^{\beta}$ the gauge invariant combination of the $S U(2)$ doublets $d_{1,2}$, then the nonperturbative superpotential is:

$$
W_{d y n}=\frac{\Lambda^{5}}{V_{12}}
$$

Because

$$
\sqrt{\alpha^{\prime}} \Lambda \sim e^{-R^{2} / \alpha^{\prime}}
$$

in terms of the radius $R$ of $C$ in the heterotic string description, we see that (2.2) is exactly the sort of superpotential one expects worldsheet instantons [6] to generate in the heterotic string theory.

${ }^{2} \mathcal{O}(n)$ denotes the line bundle with $c_{1}=n$ over $C$. 
A well known theorem of supergravity [7] requires that a superpotential contribution in an $\mathrm{N}=1$ model must have a pole at codimension one in the moduli space of vacua if it is not to vanish. 3 This theorem has been used in [8] to strongly constrain the possible superpotentials in $\mathrm{N}=1$ vacua describable as gauged linear sigma models. In more general models, however, one expects to find singular loci where (2.1) occurs: these are concrete examples of heterotic string vacua which are destabilized by worldsheet instantons. The required pole in the superpotential is none other than the pole of [1], appearing in (2.2). This agrees nicely with the observation in [8] that worldsheet instanton superpotentials can be generated when a singularity of the gauge bundle coincides with a rational curve in the manifold - in this case the relevant curve is $C$, and the singularity is the small instanton fibered over it.

\subsection{Gaugino Condensation}

Another interesting situation, which maps to a novel effect in the heterotic string, is the following. Suppose one has a model in which, at generic points in the moduli space of vacua, $V$ is singular over a point in each $K 3$ fiber. Let the splitting type of $V$ over $C$ be trivial, i.e.

$$
\left.V\right|_{C}=\mathcal{O}(0) \oplus \mathcal{O}(0) \oplus \ldots
$$

Indeed, this is the generic splitting type for a holomorphic vector bundle on $\mathbf{P}^{1}$ (with $\left.c_{1}=0\right)$. Then one expects a pure $S U(2) \mathrm{N}=1$ Yang-Mills theory to survive as the nonperturbative spectrum at the singularity, from the wrapped fivebrane analysis of [2]. One would have been uncertain about admitting that such generically singular compactifications were physically sensible before the realization that singular gauge bundles can have a concrete physical description as type I fivebranes [5]. It is now clear that such generically singular models simply correspond to $\mathrm{N}=1$ vacua where (wrapped) fivebranes are generically present.

The strong infrared gauge dynamics of the $S U(2)$ gauge group induces gaugino condensation and a superpotential

$$
W=\Lambda^{3}
$$

3 This theorem depends on compactness of the moduli space. In the cases at hand, one can compactify the moduli space by adding "points at infinity" and one knows that the relevant poles do not occur at infinity.

4 This is very similar to the realization of compact D-manifolds [9] provided by F-theory [10]. 
where $\Lambda$ is the scale of the $S U(2)$ theory. Although in global supersymmetry this would be a harmless constant contribution to the superpotential, in the string theory $\Lambda$ is dynamical (as in (2.3)) and (2.5) destabilizes the vacuum.

It is worth remarking that in the $S U(2)$ Yang-Mills theory, one can think of the superpotential (2.5) as being generated by a half-instanton effect. A choice of phase has been made in (2.5), and there are actually two vacua with $W= \pm \Lambda^{3}$, reflecting an unbroken $Z_{2}$ discrete shift symmetry of the $\theta$ angle. This unbroken $Z_{2}$ must map to an unbroken shift symmetry for the axion partner of the Kahler modulus controlling the size of $C$ in the heterotic string. Therefore, we conclude that in the heterotic string the superpotential (2.5) will appear to be a half worldsheet instanton effect!

Of course it is difficult to see how a half-instanton effect can arise from maps of the worldsheet into the compactification manifold. (2.5) arises from the two-doublet case by integrating out the massive doublets. In other words, add to (2.2) a coupling to a bundle modulus $m$ which is turned on at generic points in the moduli space where (2.4) applies. Then integrate $V_{12}$ out from the full superpotential,

$$
W_{T o t}=m V_{12}+\frac{\Lambda_{H}^{5}}{V_{12}} .
$$

One finds $V_{12}= \pm \sqrt{\frac{\Lambda_{H}^{5}}{m}}$ and $W= \pm 2 \sqrt{m \Lambda_{H}^{5}} \sim \Lambda^{3}$, reproducing (2.5). Presumably then, the sigma model on the heterotic side is computing the superpotential (2.6), which involves only integral instanton effects plus mass terms.

\subsection{Singlet Couplings and K3 Fibrations}

As discussed briefly in the previous subsection, in general we expect that varying some of the vector bundle moduli (while fixing the moduli that would remove the singularity completely) can change the splitting type of the vector bundle. For generic VEVs of bundle moduli, we expect trivial splitting (2.4), while at nonzero codimension one can obtain various nontrivial splittings, with

$$
\left.V\right|_{C}=\mathcal{O}(1) \oplus \mathcal{O}(-1) \oplus \mathcal{O}(0) \oplus \ldots
$$

5 In the case of gaugino condensation in $\mathrm{N}=1 / 0$ dual pairs obtained as orbifolds of $\mathrm{N}=2$ models [3], the origin of these effects is clear. The orbifold group is a $Z_{2}$ which acts on the base of the fibration by identifying antipodal points. Then a worldsheet can consistently wrap "half" the curve. 
the next most common. This gives rise to an $S U(2)$ gauge theory with singlet couplings to some number of $S U(2)$ doublets, where the relevant singlets are the bundle moduli which do not remove the singularity. This is precisely the structure of a model of dynamical (global) supersymmetry breaking considered by Intriligator and Thomas [11].

They considered supersymmetric QCD with $N_{F}=N_{C}$ plus singlets coupling to the flavors in such a way as to generically give mass to them. Specializing to SU(2), we have 4 doublets $d_{i}, i=1, \ldots, 4$ which combine to give gauge invariant coordinates $V_{i j}=\epsilon_{\alpha \beta} d_{i}^{\alpha} d_{j}^{\beta}$. In addition consider singlets $S^{i j}$ coupling to the other fields in the superpotential

$$
W_{I T}=S^{i j} V_{i j}+\lambda\left(\operatorname{Pf}(V)-\Lambda^{4}\right) .
$$

Integrating out $S$ in global supersymmetry, we see that supersymmetry is broken, since the point $V_{i j}=0$ has been removed from the moduli space by quantum effects. In supergravity, the condition becomes more complicated; instead of $\frac{\partial W}{\partial S}=0$, it becomes $\frac{\partial W}{\partial S}+K_{, S} W=$ 0 where $K$ is the Kahler potential. So unfortunately supersymmetry breaking is not automatic in supergravity. Also, in the string theory setting, $\Lambda$ depends on a dynamical field (the size of the $\mathbf{P}^{1}$ base of the K3 fibration). Therefore in order to fix this modulus we would require for example a product gauge group which could arise from multiple fivebranes wrapping the base (which is consistent since there is typically a family of base $\left.\mathbf{P}^{1} \mathrm{~s}\right)$.

Note that the linear term in $S$ was crucial above.6 One could also consider the situation where this is replaced by a nontrivial function $f(S)$. This would leave a supersymmetric vacuum at $f^{\prime}\left(S_{\min }\right)=0$, and the superpotential would no longer force the singularity to be frozen in.

\section{4. $E_{8} \times E_{8}$ Tensionless Strings}

One of the most interesting developments of string duality has been the realization that tensionless noncritical strings become the dominant low energy modes in some corners of moduli space [12]. In particular, this occurs in the $E_{8} \times E_{8}$ heterotic string when an instanton shrinks to zero size [13][14].

Consider the $E_{8} \times E_{8}$ string on a $K 3$ fibration $M$ with a vector bundle $V$ which develops a small instanton singularity in the generic $K 3$ fiber. On further compactification on an $S^{1}$, this theory becomes equivalent to the $S O(32)$ theories we have been studying,

\footnotetext{
6 We thank N. Seiberg for emphasizing this point.
} 
also compactified on $M \times S^{1}$. This equivalence (based on T-duality) depends on the use of Wilson lines-one must check in a given example that these are compatible with $V$.

In the cases of $\S 2.1$ and $\S 2.2$, the fibered small instanton leads to vacuum destabilization for the $S O(32)$ strings. By the equivalence mentioned in the previous paragraph, this implies that the three-dimensional $E_{8} \times E_{8}$ vacua on $M \times S^{1}$ also have no stable supersymmetry preserving vacuum. This indicates that the dynamics of the tensionless string in four dimensional $\mathrm{N}=1$ vacua can itself break supersymmetry.

As a corollary of this discussion, one also predicts that there should be a family of different $E_{8} \times E_{8}$ tensionless strings in four dimensions. For each possible splitting of $V$ on the base curve (over which it is generically singular), one should get a different tensionless string theory in four dimensions. It is important in the preceeding discussion that the $6 \mathrm{~d}$ tensionless strings are effectively being compactified on $C$ which has $\pi_{1}(C)=0$. This means that there are no winding modes of the tensionless strings (which would have appeared as particles in four dimensions), so nothing will replace the noncritical string as the most relevant low energy degree of freedom.

\section{F-theory Examples}

The discussion in section 2 was general; it is of obvious interest to get control over specific examples of the above phenomena. The $\mathrm{N}=1$ heterotic models that have been the focus of previous studies have been realized as gauged linear sigma models on the worldsheet [15] [16]. The superpotential vanishes in such models, so the conditions under which destabilization is possible are very limited [8].7 One way to construct more generic models, which do admit the phenomena of $\S 2$, is to use the duality between heterotic models and F-theory (as was done in [17]).

One can derive F-theory/heterotic dualities for four-dimensional $\mathrm{N}=1$ theories by starting with the accepted dualities in higher dimensions [10 and applying the adiabatic argument. This leads one to believe that F-theory compactified on an elliptic fourfold

7 Destabilization would require the presence of a "twisted singlet" (i.e. a singlet whose VEV would describe a deformation away from the linear sigma model moduli space) with linear coupling to the other singlets in a nontrivial superpotential with the correct singularity structure.

8 For a discussion of an analogous question of superpotentials on 3-branes in six dimensions, where it is relatively simple to construct examples as gauged linear sigma models on the heterotic side, see [18]. 
$X$ which has as base a rationally ruled threefold $B=\mathbf{P}^{1} \rightarrow B^{\prime}$ should be dual to some heterotic string compactification on the elliptically fibered Calabi-Yau $Y=T^{2} \rightarrow B^{\prime}$. Discussions of such dualities have appeared recently in [17] [19] [20].

One peculiar feature of these compactifications is that the F-theory side requires the presence of $\chi(X) / 24$ threebranes for consistency. These must map to fivebranes, wrapped around the $T^{2}$, in the dual heterotic compactification on $Y$. In this section, we will be using purely local considerations which should not be affected by the presence of such branes - we can move them away from the relevant singular locus so our arguments should be valid also in cases when no branes are present.

In the following two subsections we will present examples of F-theory compactifications on elliptic fourfolds $X_{1}$ and $X_{2}$ which are dual, by the adiabatic argument, to type I/heterotic compactifications on threefolds $Y_{1,2}$ which exhibit the phenomena of $\S 2.1$ and $\S 2.2$

\subsection{An Example of Instanton Destabilization}

The fourfold $X_{1}$ can be described as follows. It is given as a hypersurface in a toric variety which is of the rough form $C^{9} /\left(C^{*}\right)^{4}$. There are homogeneous coordinates $r, s, u, v, p, t, x, y, q$ and the $C^{*}$ symmetries $(\lambda, \mu, \nu, \rho)$ act on these coordinates with exponents given in the table below:

\begin{tabular}{|c|c|c|c|c|c|c|c|}
\hline$r$ & $s$ & $u$ & $v$ & $p$ & $t$ & $x$ & $y$ \\
\hline 1 & 1 & 6 & 0 & 16 & 0 & 48 & 72 \\
\hline 0 & 0 & 1 & 1 & 4 & 0 & 12 & 18 \\
\hline 0 & 0 & 0 & 0 & 1 & 1 & 4 & 6 \\
\hline 0 & 0 & 0 & 0 & 0 & 0 & 2 & 3 \\
\hline
\end{tabular}

This manifold is a fibration of the elliptic fibration over $F_{4}$ over an additional $\mathbf{P}^{\mathbf{1}}$. Because the elliptic fibration over $F_{4}$ is dual in six dimensions to the type I theory [10], this fourfold should be dual by the adiabatic argument to some $\mathrm{N}=1$ compactification of the $S O(32)$ strings. In fact, since the base of the elliptic fibration $B$ is a $\mathbf{P}^{\mathbf{1}}$ fibration over $F_{6}$, the F-theory compactification should be dual to a heterotic/type I compactification on the elliptic fibration over $F_{6}$.

9 As explained in [19, it is not clear what the precise situation is with regard to the need for threebranes in cases when $X$ is generically singular, which are the cases of interest for us. 
$X_{1}$ has the form of a fibration $T^{2} \rightarrow \mathbf{P}^{\mathbf{1}} \rightarrow \mathbf{P}^{\mathbf{1}} \rightarrow \mathbf{P}^{\mathbf{1}}$, where the coordinates on the three $P^{1} \mathrm{~s}$ are

$$
z_{1}=r / s, \quad z_{2}=v / u, \quad z_{3}=p / t
$$

One can imagine taking the $\mathbf{P}^{\mathbf{1}}$ with homogeneous coordinates $r, s$ to be very large. In this limit, one obtains a six-dimensional theory dual to the type I compactification on $K 3$.

As we will show below, at complex codimension one in the moduli space of vacua, one can see that $X_{1}$ develops an $I_{2}$ singularity (in Kodaira's classification) at $v=0$ which looks (in the six-dimensional limit) like the F-theory description of a single small instanton [21] 222], and gives rise to a nonperturbative $S U(2)$ gauge group. Upon further fibration to four dimensions on the last $\mathbf{P}^{\mathbf{1}}$, which we call $C$, this yields an F-theory model dual to a type I/heterotic theory which develops a small instanton in the generic $K 3$ fiber at codimension one. This is equivalent to a type I vacuum with a D 5-brane wrapped around C.

In addition to the nonperturbative $S U(2)$ gauge group, some of the charged matter associated to the D 5-brane must also survive the reduction to four dimensions. Since the singularity is at complex codimension one, the simplest possibility is that precisely two doublets $d_{1,2}$ survive the reduction. We will argue that this is the case below. Hence, we will find ourselves with an example of precisely the situation described in $\S 2.1$.

These results are most evident if one examines the Weierstrass form for $X_{1} \cdot X_{1}$ is defined by an equation

$$
y^{2}=x^{3}+q^{4} x \sum_{i=0}^{6} p^{i} t^{8-i} f_{96-16 i, 24-4 i}\left(z_{1}, z_{2}\right)+q^{6} \sum_{j=0}^{9} p^{j} t^{12-j} g_{144-16 j, 36-4 j}\left(z_{1}, z_{2}\right)
$$

where the subscripts on the $f \mathrm{~s}$ and $g$ s indicate the charges under the first two $C^{*}$ actions.

Expanding $f$ and $g$ in $v$, we find

$$
\begin{aligned}
& f \sim a_{0} p^{6} t^{2} q^{4}+a_{2} p^{5} v^{2} u^{2} t^{3} q^{4} H_{4}(r, s) \\
& g \sim b_{0} p^{9} t^{3} q^{6}+b_{2} p^{8} v^{2} u^{2} t^{4} q^{6} \tilde{H}_{4}(r, s)
\end{aligned}
$$

where $H_{4}(r, s)$ and $\tilde{H}_{4}(r, s)$ are fourth-degree polynomials in $r$ and $s$, the coordinates on the base $\mathbf{P}^{1}$. To understand the singularity structure, we must plug these into the equation

$$
\Delta=4 f^{3}+27 g^{2}
$$


for the discriminant. Then we see that for

$$
\begin{gathered}
4 a_{0}^{3}=-27 b_{0}^{2}, \\
\Delta \sim v^{2} t^{7}\left(H_{4}(r, s)+\tilde{H}_{4}(r, s)\right) .
\end{gathered}
$$

Thus at the codimension one locus (3.6), there is a Kodaira type $I_{2}$ singularity, corresponding to enhanced $S U(2)$ gauge symmetry. Since the singularity is localized in $z_{2}=v / u$, this gauge symmetry enhancement is nonperturbative on the heterotic side [21] [22].

It is important to understand not only the codimension of the singularity, but the precise matter content charged under the enhanced $S U(2)$ that survives in four dimensions. There is no direct correlation between the codimension and the matter content in $4 d N=1$ supersymmetry because of the possibility of a tree-level superpotential.

Let us pause to explain this in more detail. In six dimensions, each small instanton is equipped with matter in the $(\mathbf{3 2}, \mathbf{2})$ of $S O(32) \times S U(2)$. Upon reduction on the base $\mathbf{P}^{1}$, the $4 d$ spectrum will contain some number $n_{d}$ of doublets; others will develop Kaluza-Klein masses. The surviving $n_{d}$ doublets could couple in a nontrivial tree level superpotential. Generically this would start at fourth order in the doublets, or at quadratic order in the gauge invariant coordinates $V_{i j}=d_{i}^{\alpha} d_{j}^{\beta} \epsilon_{\alpha \beta}$; away from the singularity, these contributions would look like mass terms for singlets. Therefore codimension alone is not a reliable guide to the physics. In order to determine the presence of a dynamically generated superpotential, one needs at least two of three classical inputs: charged matter spectrum, codimension of the singularity, and tree-level superpotential. In the previous study [2], we computed the first two, which determined enough of the third to proceed to an understanding of $W_{d y n}$. In the present case, we have a handle only on the codimension of the singularity.

In this case we can, however, determine the presence of the expected dynamical superpotential by invoking the analysis of [17]. This will also provide a connection between the gauge theory cases we are studying here and the discussion provided there. In [17], it was shown that contributions to the superpotential in F-theory compactification on $X_{1}$ correspond to divisors $D$ in the fourfold with $\chi\left(D, \mathcal{O}_{D}\right)=1$, where

$$
\chi\left(D, \mathcal{O}_{D}\right)=h^{0,0}(D)-h^{1,0}(D)+h^{2,0}(D)-h^{3,0}(D)
$$


is the arithmetic genus of $D$. Furthermore, the superpotential will correspond to a worldsheet instanton effect on the heterotic side if the divisor $D$ involves only a curve in the last two $\mathbf{P}^{1} \mathrm{~s}$ (the part of the F-theory manifold that is visible on the heterotic side). 10

In our example, there is an obvious such divisor $D$ :

$$
v=0
$$

That this divisor has $\chi\left(D, \mathcal{O}_{D}\right)=1$ can be seen as follows (following $\S 2.1$ of [17]). First note that since $v$ is the only polynomial in the projective coordinates with charges $(0,1,0,0)$, there are no deformations of this divisor. Hence, $h^{3,0}(D)=0$. One can then use the Lefschetz hyperplane theorem to fix $h^{1,0}(D)$ and $h^{2,0}(D)$. This theorem states that for $D$ a divisor in $M$ determined by the vanishing of a section of a positive line bundle on $M$, there is an isomorphism between the integral cohomology of $M$ and that of $D$, up to (but not including) the middle dimension. In our case, since it has positive charge, $v$ is a section of a positive line bundle, so the theorem applies to the divisor (3.9). Similarly, the theorem applies to the Calabi-Yau fourfold $X_{1}$. Since $h^{1,0}\left(X_{1}\right)=h^{2,0}\left(X_{1}\right)=0$ follows from the fact that $X_{1}$ is simply connected and has $S U(4)$ holonomy instead of a proper subgroup, we learn that our divisor (3.9) also has $h^{1,0}(D)=h^{2,0}(D)=0$. Putting it together, the only contribution to $\chi\left(D, \mathcal{O}_{D}\right)$ is from $h^{0,0}(D)$, yielding $\chi\left(D, \mathcal{O}_{D}\right)=1$.

Therefore the locus (3.9) determines a contribution to the superpotential, which moreover corresponds to a worldsheet instanton on the heterotic side wrapping the base of the $K 3$ fibration $Y_{1}=T^{2} \rightarrow F_{6}$. Let us denote the Kahler modulus of this $\mathbf{P}^{1}$ as $T$. The superpotential will contain, in addition to the classical piece $e^{-T}$, holomorphic dependence on the other moduli. As discussed in [7], the superpotential must have a pole at codimension one on the space of chiral superfields, assuming that space is compact. Therefore, there must be codimension one singularities in F-theory at which the superpotential can diverge. In our problem, we have just this structure. As explained above, at codimension one in the moduli space of vacua, the elliptic fiber has an $A_{1}$ degeneration on the divisor (3.9). So we have a superpotential at the one worldsheet instanton level which blows up at the locus with enhanced non-perturbative $S U(2)$. This is just the situation discussed in $\S 2.1$ for $S U(2)$ with two doublets.

10 The results of [17] were derived and applied in the context of models without generic singularities. The model under present discussion has a generic singularity (related to a perturbative heterotic gauge group) at $z_{3}=\infty$, which is irrelevant to the physics we are discussing. We are assuming that this extra singularity does not invalidate our use of the results of [17]. 


\subsection{An Example of Gaugino Condensation}

We can similarly present an F-theory compactification on a fourfold $X_{2}$ which is dual to the situation envisioned in $\S 2.2$, though with somewhat less control given the generic singularity and a strange feature which will soon become apparent. The toric data for $X_{2}$ is given in the table below:

\begin{tabular}{|c|c|c|c|c|c|c|c|c|}
\hline & $r$ & $s$ & $u$ & $v$ & $p$ & $t$ & $x$ & $y$ \\
\hline$\lambda$ & 1 & 1 & 3 & 0 & 11 & 0 & 32 & 48 \\
\hline$\mu$ & 0 & 0 & 1 & 1 & 4 & 0 & 12 & 18 \\
\hline$\nu$ & 0 & 0 & 0 & 0 & 1 & 1 & 4 & 6 \\
\hline$\rho$ & 0 & 0 & 0 & 0 & 0 & 0 & 2 & 3 \\
\hline
\end{tabular}

$X_{2}$ is a hypersurface in this toric variety with Weierstrass form

$$
y^{2}=x^{3}+q^{4} x \sum_{i=0}^{5} p^{i} t^{8-i} f_{64-11 i, 24-4 i}\left(z_{1}, z_{2}\right)+q^{6} \sum_{j=0}^{8} p^{j} t^{12-j} g_{96-11 j, 36-4 j}\left(z_{1}, z_{2}\right)
$$

with the same notation as in (3.2). By the adiabatic argument, it should be dual to a type I/heterotic compactification on $Y_{2}=T^{2} \rightarrow F_{3}$.

It follows from the data defining $X_{2}$ that none of the $f \mathrm{~s}$ can contain a term independent of $z_{2}$ (i.e. proportional to $v^{0}$ ) and only one, $f_{9,4}$, can contain a linear term in $z_{2}$ (which is then independent of the other coordinates). Similarly, none of the $g$ s can contain constants or terms linear in $z_{2}$, but quadratic terms in $z_{2}$ are allowed. This means that the discriminant is of the form

$$
\Delta=z_{2}^{3} t^{9}\left(\mathrm{const}+O\left(z_{2}\right)\right)
$$

expanded around $z_{2}=0$. From [10] 222 one sees that this is a type III singularity in Kodaira's classification, which yields an $S U(2)$ gauge group. This means that there is a small instanton in the generic $K 3$ fiber (fibered over the $\mathbf{P}^{1}$ with coordinate $z_{1}$ ) in the dual heterotic string description. 11

11 It is actually an $I_{2}$ singularity at $z_{2}=0$ which was found to be dual to generic small instantons in [21] 22]. The type III singularity is to be physically thought of as a special (more degenerate) case of $I_{2}$ which still yields the same gauge group. Imposing type III instead of $I_{2}$ gives a proper submoduli space of the small instanton moduli space . 
Similarly to the case of $X_{1}$, here we have a divisor $v=0$ with no deformations. Again the Lefschetz theorem applies, and using the isomorphism of the cohomology to that of the fourfold, $\chi\left(D, \mathcal{O}_{D}\right)=1$ and there will be a superpotential. Here the locus $v=0$ is a frozen in singularity, so we expect the superpotential to diverge. This is consistent with the following superpotential:

$$
W=A V_{12}^{k}+\frac{\Lambda^{5}}{V_{12}}
$$

for some $k$ (the presence of the first term is necessitated by the fact that the singularity is frozen in). If $k=1$ we recover the gaugino condensation superpotential of $\S 2.2$. Unfortunately, this is difficult to determine here. In any case, integrating out $V_{12}$ gives a superpotential which has fractional instanton number.

There are some strange features of this model which make its dual interpretation as a type I vacuum somewhat hazy. In particular, in addition to the small instanton singularity, the gauge symmetry at $z_{3}=\infty$ has been enhanced to $F_{4}$. While this $F_{4}$ can be described

as a perturbative gauge symmetry in the $E_{8} \times E_{8}$ heterotic string dual [10][21] 22] (which one would obtain by fibering the $E_{8} \times E_{8}$ string on $K 3$ with $(16,8)$ instantons in the two $E_{8}$ s over the last $\mathbf{P}^{\mathbf{1}}$ ), it is not a symmetry which one expects to arise perturbatively in the type I or $S O(32)$ heterotic string description.

If this subtlety with the gauge group at $z_{3}=\infty$ does not affect the interpretation of the $A_{1}$ singularity, then this F-theory model is (dual to) an example of the sort described in $\S 2.2$ : A small instanton in the generic $K 3$ fiber of the type I / heterotic compactification has yielded a pure $S U(2)$ gauge group in four dimensions. Hence, the vacuum is destabilized by gaugino condensation / half worldsheet instantons.

\section{Conclusions}

Many supersymmetric $S O(32)$ string compactifications, including those with no interesting dynamics originating from perturbative gauge groups, should be lifted by nonperturbative effects originating in nonperturbative gauge groups. While the examples of $\S 3$ were given in the framework of F-theory, it is clear that the phenomena of $\S 2$ are far more general and will occur in heterotic models without any (at least presently known) candidate F-theory dual.

It would be interesting to explore heterotic/type I models with such nonperturbative superpotentials more directly, perhaps using techniques appearing in [18]. 
A complementary approach to the study of $\mathrm{N}=1$ models with superpotentials will appear in [23], where a particular type I orbifold is studied in great detail.

\section{Acknowledgements}

We would like to thank T. Banks, J. Distler, D. Morrison, C. Vafa, and especially N. Seiberg for helpful discussions. This work was completed while the authors were enjoying the hospitality of the Aspen Center for Physics. 


\section{References}

[1] I. Affleck, M. Dine, and N. Seiberg, "Dynamical Supersymmetry Breaking in Supersymmetric QCD", Nucl. Phys. B241 (1984) 493.

[2] S. Kachru, E. Silverstein, and N. Seiberg, "SUSY Gauge Dynamics and Singularities of 4d N=1 String Vacua," hep-th/9605036.

[3] S. Kachru and E. Silverstein, "N=1 Dual String Pairs and Gaugino Condensation", Nucl. Phys. B463 (1996) 369, hep-th/9511228.

[4] E. Witten, "New Issues for Manifolds of SU(3) Holonomy," Nucl. Phys. B268 (1986) 79 .

[5] E. Witten, "Small Instantons in String Theory," Nucl. Phys. B460 (1996) 541, hepth/9511030.

[6] M. Dine, N. Seiberg, X. G. Wen, E. Witten, "Nonperturbative Effects on the String Worldsheet", Nucl. Phys. B278 (1986) 769; Nucl. Phys. B289 (1987) 319.

[7] E. Witten and J. Bagger, "Quantization of Newton's Constant in Certain Supergravity Theories", Phys. Lett. B115 (1982) 202.

[8] E. Silverstein and E. Witten, "Criteria for Conformal Invariance of $(0,2)$ Models", Nucl. Phys. B444 (1995) 161.

[9] M. Bershadsky, V. Sadov, and C. Vafa, "D strings on D manifolds", Nucl. Phys. B463 (1996) 398, hep-th/9510225.

[10] C. Vafa, "Evidence for F-theory," hep-th/9602022;

D. Morrison and C. Vafa, "Compactifications of F-theory on Calabi-Yau Threefolds I, II," hep-th/9602114,9603161.

[11] K. Intriligator and S. Thomas, "Supersymmetry Breaking on Quantum Moduli Spaces", hep-th/9603158.

[12] E. Witten, "Some Comments on String Dynamics", hep-th/9507121.

[13] O. Ganor and A. Hanany, "Small E(8) Instantons and Tensionless Noncritical Strings", hep-th/9602120.

[14] N Seiberg and E Witten, "Comments on String Dynamics in Six Dimensions", Nucl. Phys. B471 (1996) 121, hep-th/9603003.

[15] E. Witten, "Phases of N=2 Theories in Two Dimensions", Nucl. Phys. B403 (1993) 159, hep-th/9301042

[16] J. Distler and S. Kachru, "(0,2) Landau-Ginzburg Theory", Nucl. Phys. B413 (1994) 213, hep-th/9309110.

[17] E. Witten, "Nonperturbative Superpotentials in String Theory," hep-th/9604030.

[18] J. Distler, S. Kachru, and E. Silverstein, to appear.

[19] S. Sethi, C. Vafa, E. Witten, "Constraints on Low-Dimensional String Compactification", hep-th/9606122.

[20] I. Brunner and R. Schimmrigk, "F Theory on Calabi-Yau Fourfolds", hep-th/9606148. 
[21] P. Aspinwall and M. Gross, "The $\mathrm{SO}(32)$ Heterotic String on a K3 Surface", hepth/9605131.

[22] M. Bershadsky, K. Intriligator, S. Kachru, D. Morrison, V. Sadov, and C. Vafa, "Geometric Singularities and Enhanced Gauge Symmetry," hep-th/9605200.

[23] M. Berkooz and R. Leigh, "A D=4 N=1 Orbifold of Type I Strings", hep-th/9605049 and to appear. 